\title{
A cross-modal interference effect in grasping objects
}

\author{
SANDHIRAN PATCHAY and UMBERTO CASTIELLO \\ Royal Holloway University of London, Egham, England \\ and \\ PATRICK HAGGARD \\ University College London, London, England
}

\begin{abstract}
The aim of the present paper was to investigate how the kinematics of a hand reaching toward a visual target would be influenced by haptic and proprioceptive input from an unseen distractor actively grasped in the other, nonreaching hand. The main results were that the amplitude of maximum grip aperture was smaller and the time to maximum grip aperture was earlier when the distractor was smaller than the target. The interference effect from the distractor was similar for both hands as they reached. Furthermore, results from a vibrating-distractor condition for passive tactile input revealed that the interference effects were evident only when the distractor was actively grasped. We suggest that neural processing of proprioceptive and tactile information relevant to distractor size produced the observed interference effects. We also emphasize the importance of active manipulation of the distractor stimulus in eliciting such interference effects.
\end{abstract}

To further our understanding of perception and action, recent studies have examined reach-to-grasp tasks that involved responding to a visual target in the presence of visually distracting objects within a three-dimensional (3-D) space (Castiello, 1996; for reviews, see Bonfiglioli \& Castiello, 1998, Castiello, 1999, and Tipper, Howard, \& Jackson, 1997). For example, Tipper et al. found that the presence of a distractor could affect the trajectory of the reaching movement to a target. In a study requiring participants to reach and grasp a target while simultaneously attending to a distractor, Castiello (1996) reported that the maximum grip aperture was greater when the distractor was larger than the target and smaller when the distractor was smaller than the target. Such differences were not found when the same distractors were present but not attended to. As has been suggested by Deubel, Schneider, and Paprotta (1998), with higher levels of attentional focus, the volumetric features of a distractor appear to influence action pathways. However, these reach-to-grasp studies on distractor interference have focused on visual inputs, without considering the possibility of cross-modal distractor interference.

The notion that senses are better conceptualized as interrelated modalities, rather than as independentchannels,

S.P. gratefully acknowledges the support of Fondation Singer Polignac, Paris, and The Wellcome Trust. The authors are grateful to Claudia Bonfiglioli for her help with the Elite system and to James Taylor, Andy Haswell, Mark Wells, and the late Derek Neil for technical assistance. Correspondence concerning this article should be addressed to S. Patchay, Department of Psychology and Counselling, School of Health and Social Care, University of Greenwich, Avery Hill Campus, Southwood Site, Avery Hill Road, Eltham, London SE9 2UG, England (e-mail: s.patchay@gre.ac.uk). has recently been supported by several studies that have provided evidence for common neural and attentional mechanisms in the processing of multisensory information (see, e.g., Doyle \& Walker, 2002; Driver \& Spence, 1999; Graziano \& Gross, 1993; Jolicœur, 1999; Spence, Pavani, \& Driver, 2000). In these studies, the emphasis has been on cross-modal links between combinations of visual, auditory, and tactile inputs. A common finding has been that better responses were elicited when the stimuli were located in the same (or a very close) position, rather than in different positions, in external space.

Most of the research conducted with humans on crossmodal integration has typically focused on perceptual integration and has studied this by assigning arbitrary responses (e.g., reaction time or saccadic eye movements). The effects of multisensory coding during more natural tasks, such as upper limb tasks requiring action in 3-D space, are understood less well. An attempt to investigate the issue of cross-modal links between haptic and visual information when a natural reach-to-grasp task is performed has been provided by Gentilucci, Daprati, and Gangitano (1998). In their experiments, participants reached and grasped a visual target (sphere) presented in different sizes with one hand, while holding another, unseen sphere (distractor) of different sizes in the other hand. These authors found that manipulation with the right hand influenced finger shaping of the grasping left hand when the two objects differed in size. They reported an interference effect only for the unseen small distractor object, but with only an effect of the right hand on the left hand. However, in their study, it is unclear what caused the interference. They argued that haptic information reaching one hemisphere 
had an interference effect on the other hemisphere but that the left hemisphere acquired more precise information and induced a greater effect.

It is known that peripheral mechanoreceptors, including proprioceptors (muscle spindles and joint receptors) and cutaneous receptors in the glabrous skin, are engaged during active digit movements in man (Burke, Gandevia, \& Macefield, 1988; Johansson \& Cole, 1994). Tactile and proprioceptive information from the hand primarily reaches the contralateral primary somatosensory (S1) cortex and can secondarily reach the ipsilateral side via corticocortical connections (Ghez \& Krakauer, 2000). The S1 cortex is a major source of input to the motor cortex and, as such, has direct access to the primary motor cortex. Descending influence to effectors is then achieved contralaterally through the lateral corticospinal tracts and bilaterally through the ventral tract. According to this anatomical and functional evidence, there is, then, good reason to believe that similar effects can also be expected for both hands.

In the present study, our aim was to investigate how proprioceptive and haptic information from a distractor stimulus actively grasped in one hand can influence the reaching and grasping components of the other hand when it is reaching toward a visual target. Most important, we also seek to demonstrate how purely proprioceptive information may affect visually guided action under the right conditions.

We used a reach-to-grasp interference paradigm featuring the following. (1) Tactile and proprioceptive information from the distractor was available to one hand before the signal was given to start the reach-to-grasp movement toward the target with the other hand. This procedure was followed because there is evidence suggesting that proprioceptive afferents play a significant role, along with cutaneous afferents, in the perception of hand conformation (Cordo, Carlton, Bevan, Carlton, \& Kerr, 1994; Johnson, Yoshioka, \& Vega-Bermudez, 2000). If proprioceptive and haptic information from the distractor is made available, then, in line with the findings of these authors, we would expect interference of this information on the kinematics of the hand reaching toward the target. (2) Both hands performed the same type of action-that is, the grasping action of the hand required to close upon the distractor was similar to that of the other hand grasping the target. This constitutes a major difference with the experiment of Gentilucci et al. (1998), in which the participant passively manipulated the distractor object from beneath it. Their protocol required that the hand be constrained in a particular posture to perform the manipulating action, employing a motor program different from that used for reaching and grasping the target. Hence, it is likely that the interference effect reported by Gentilucci et al. might have originated from the activation of two concurrent separate motor programs for the two hands, rather than from the size of the distractor itself. Therefore, if the same type of action is performed employing the same motor program, we can exclude any effect from competition between two different motor programs. (3) Another differ- ence between the present experiment and that of Gentilucci et al. is that the target and the distractor locations were coincident. It has already been reported that enhanced overt attentive and orientation responses are induced when two different sensory stimuli are spatially concordant (Graziano \& Gross, 1993; Spence et al., 2000; Stein, 1998). If target and distractor locations are spatially concordant, we would expect that interference from the distractor would be evident from the target kinematics. (4) Finally, we included a vibrating condition that allowed a crucial comparison with the distractor conditions. The importance of movement to touch is underlined by the observation that tactile perception is better when there is movement between the stimulus and the skin (active touch), as compared with when the stimulus is applied passively (Chapman, 1994). The aim of this condition was to determine whether any interference was due simply to the tactile input from the distractor or, alternatively, whether the distractor tactile input had to be part of a complete objectoriented action.

\section{METHOD}

\section{Participants}

Eight healthy right-handed volunteers ( 5 males and 3 females, 21-30 years of age), recruited from the university student population, took part in the study. All the participants had normal or correctedto-normal vision, and none of them had any known neuromuscular disorder affecting the upper limbs. All of them were naive as to the purpose of the experiment, and in compliance with the Royal Holloway University of London Ethical Committee regulations, written informed consent was obtained from each one prior to his/her inclusion in the study. Each participant attended one experimental session of 1-h duration.

\section{Material and Apparatus}

The experimental setup (see Figure 1) was displayed in a well-lit room. The participants were seated comfortably facing the work surface. The stimuli were two identical inflatable rubber bulbs (height, $9 \mathrm{~cm}$ ). One stimulus (the target) was positioned at the center of the work surface (depth, $44 \mathrm{~cm}$; width, $42 \mathrm{~cm} ; 100 \mathrm{~cm}$ from ground level). The target had a constant diameter of $5.5 \mathrm{~cm}$, and it was filled with an epoxy compound to render it unalterable when grasped. The other stimulus (the distractor) was located in a position coincident with the target $86 \mathrm{~cm}$ from ground level in a compartment opened at both ends-that is, underneath the work surface (see Figure 1). The distractor was inflated and deflated by a computer-controlled pneumatic apparatus. Customized software regulated the passage of compressed air (maximum pressure, $138 \mathrm{kPa}$ ) to the bulb. The airflow was appropriately timed to inflate the distractor stimulus to the required size. A one-way valve enabled air to be locked in the system with minimum leakage, thus maintaining the required size of the distractor constant throughout a trial. Another valve was activated to release the air and deflate the bulb. The participant could not see the distractor during the experiment.

The participant's hands were positioned as follows. The hand (right or left, depending on condition) required to reach toward and grasp the target (target hand) rested on the starting switch, with the index finger and the thumb held in slight opposition and the more ulnar digits flexed. The starting switch was flush with the work surface and was positioned $22 \mathrm{~cm}$ from the target's center and $8 \mathrm{~cm}$ from the participant's thorax. The hand (right or left, depending on condition) required to close upon the distractor (distractor hand) was 


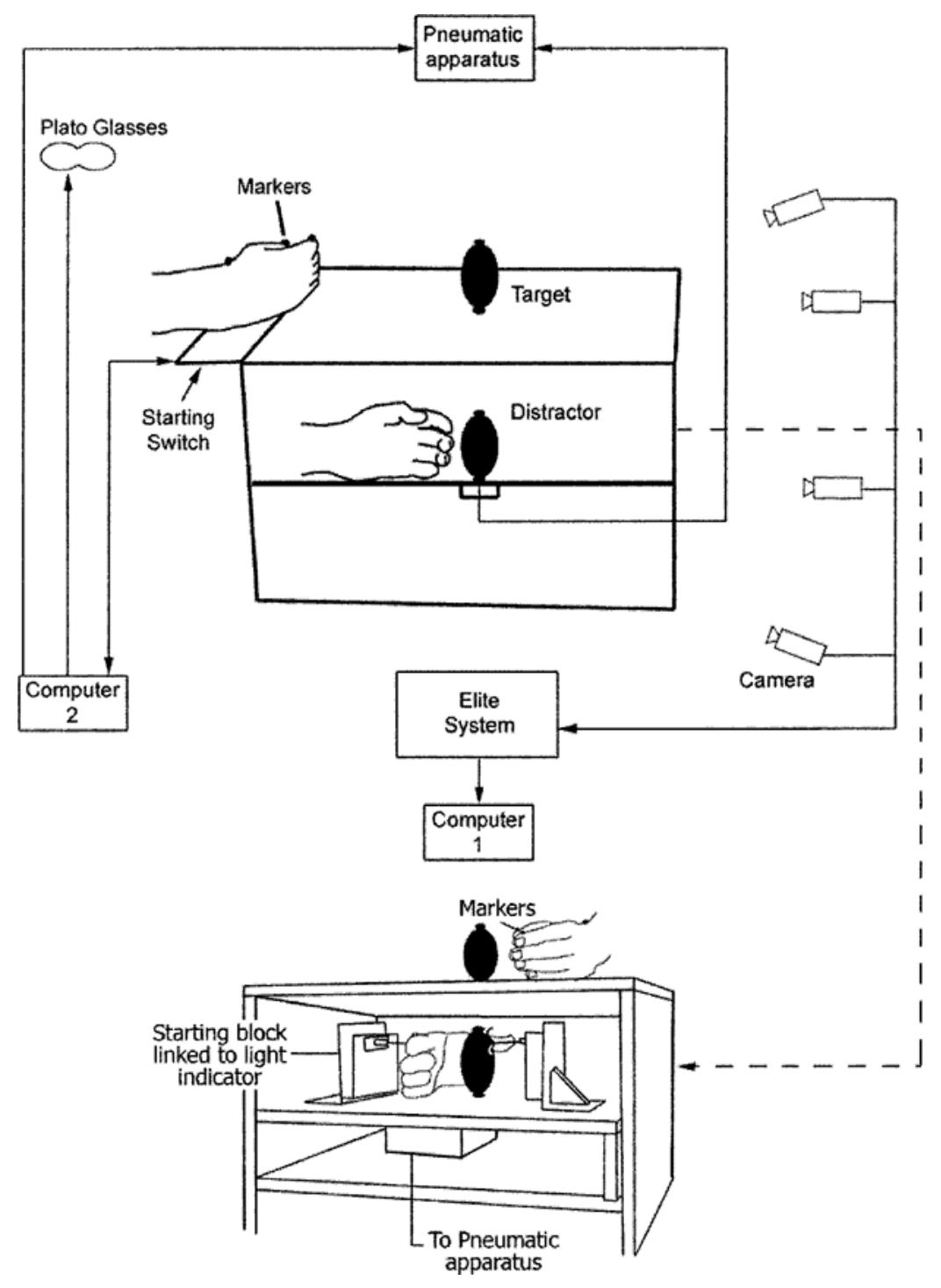

Figure 1. Schematic representation of the experimental setup (see the text for a detailed description): transverse view (upper panel) and front view (lower panel) of the work surface, showing the spatially coincident locations of the target and the distractor and the starting positions of both hands.

positioned so that the thumb and the index finger rested in adjustable starting blocks, mounted on contact microswitches positioned on the lateral and anterior sides of the distractor. This ensured that the participant's index finger and thumb were held in a consistent starting position (open grip) before the start of each trial. A light indicated to the experimenter the correct starting position of the distractor hand. Furthermore, the distractor was connected to an oscillator. The oscillator was driven by a $16-\mathrm{Hz}$ square wave signal fed through an amplifier at $5 \mathrm{~V}$, sufficient to provide a clearly suprathreshold vibrating signal. This computer-controlled nonnoxious mechanical vibration was parallel to the vertical axis of the distractor and was used in a vibrating-distractor task (see below) in our experiment.

During the whole experiment, the participants wore lightweight spectacles fitted with liquid crystal lenses (Plato Technologies Inc.) that rendered the target stimulus visually accessible by changing from opaque to clear. The lenses cleared instantly ( $1 \mathrm{msec})$, so that no delay in the reaching movement was engendered when the spectacles changed from opaque to clear. The same customized software operated the clearing of these lenses.

\section{Data Acquisition}

Kinematics of the target hand were recorded with and processed by a four-camera Elite motion analysis system (BTSwin, Milan, Italy). The system was used to collect 3-D time displacement data from three infrared-reflecting hemispherical passive markers $(\varnothing, 6 \mathrm{~mm})$. The markers were positioned on the wrist (radial aspect of the distal styloid process of the radius), the index finger (radial side of the nail), and the thumb (ulnar side of the nail). The displacement of the markers was recorded with four $100-\mathrm{Hz}$ video cameras. Two cameras were positioned $2.7 \mathrm{~m}$ in front of the participant at a $30^{\circ}$ intracam- 
era angle, and the other two cameras were placed $1.5 \mathrm{~m}$ frontolaterally on the right and left sides of the participant at a $90^{\circ}$ intracamera angle. The calibrated working space was $50 \mathrm{~cm}$ deep, $40 \mathrm{~cm}$ high, and $30 \mathrm{~cm}$ wide, from which the spatial accuracy measured from stationary and moving stimuli was $0.3 \mathrm{~mm}$.

\section{Procedure}

Three tasks were administered and are described below.

Target alone task-baseline condition. In this task, only the target was presented, and this constituted our baseline control condition (BC). The participant had to reach and grasp the target with the index finger and thumb at normal speed as soon as the lenses of the spectacles cleared. The lenses stayed clear for 3,000 msec. The participant was further instructed to keep holding the target for a little while before coming back to the starting position, ready for the next trial. This baseline condition was administered at the outset of the study, so that the participant had no prior exposure to the conditions in which the distractor stimulus was introduced. Each participant performed a set of 16 reaches with each hand. A short period of rest was included between each set.

Vibrating distractor task. A vibrating distractor condition (VC) was presented in the second task. For this task, the diameter of both the target and the distractor was $55 \mathrm{~mm}$. However, the size of the vibrating distractor was not relevant to the task. The participant was instructed to feel the vibration in one hand throughout the trials without grasping the distractor. The other hand reached and grasped the target each time the lenses cleared. Each participant completed a set of 16 reaches with each hand; a short period of rest was included between each set. The purpose of this task was to give the participant a continuous and passive proprioceptive input to be compared with the distractor task (see below).

Distractor task. In this task, one hand grasped the distractor while the other hand reached and grasped the target. Four conditions were administered. These conditions were labeled as follows: (1) the nodistractor control condition ( $\mathrm{C} 0)$, in which the target alone was presented, a condition similar to the baseline condition; (2) the small distractor condition $(\mathrm{C} 1)$, in which the distractor diameter was $43 \mathrm{~mm}$ and the target diameter was $55 \mathrm{~mm}$; (3) the same-size distractor condition $(\mathrm{C} 2)$, in which the diameter of both the target and the distractor was $55 \mathrm{~mm}$; and (4) the large distractor condition (C3), in which the target diameter was $55 \mathrm{~mm}$ and the distractor diameter was $75 \mathrm{~mm}$. These conditions occurred in random order and were presented in equal numbers within each set of trials. For these conditions, the sequence of events was the following. The experimenter triggered each trial from the computer keyboard. Upon the pressing of a key, the distractor was inflated according to the condition. Upon hearing a tone $(1000 \mathrm{~Hz}, 500 \mathrm{msec})$ delivered $1,000 \mathrm{msec}$ after the lenses turned opaque, the participant had to close the hand on the distractor. Then the lenses cleared at irregular intervals between 1,000 and $2,000 \mathrm{msec}$ after the tone, and the participant was instructed to reach and grasp the target at normal speed as soon as the lenses cleared and to remain holding the target for a little while. In the no-distractor condition, no tone was delivered; when the lenses cleared, the participant had only to reach and grasp the target while keeping the index finger and thumb of the distractor hand on the starting blocks. After the prehension movement had been completed, the starting position for each hand was resumed for the next trial. Contact with the distractor was maintained until the end of every trial. Each participant completed four sets of 16 trials with each hand. The order of hands was counterbalanced across participants. Practice trials were performed before the experiment, and a short period of rest was allowed after each set of trials.

\section{Data Processing}

The BTSwin software package was used to construct the 3-D coordinates of the markers from the images. A morphological model of the hand (grip) was used to reconstruct the location of the mark- ers and their links. The resulting $x$-, $y$-, and $z$-axis displacement data were then smoothed using a low-pass finite impulse response linear filter with an automated cutoff frequency (D'Amico \& Ferrigno, 1992).

The statistical analyses have been confined to the dependent variables that were thought to be specifically relevant to the scientific hypothesis under test. For the reaching component, the variables of interest were movement duration and the deceleration time (time from peak velocity to the end of the movement). For the grasp component, these variables were the amplitude of maximum grip aperture and the time at which this maximum aperture occurred (time of maximum grip aperture). The amplitude of maximum grip aperture refers to the maximum distance between the two markers positioned on the index finger and the thumb. This value was expressed as a percentage of maximum opening of the participant's thumb and index finger (measured with a ruler), in order to have comparable data between different hand sizes. Note that the statistical patterns of the normalized and absolute values of those amplitudes were found to be identical.

These variables were chosen because consistent results within the reach-to-grasp literature have shown that movement duration and deceleration time are longer for small than for large objects. For the grasp component, the amplitude of maximum grip aperture is reduced and is reached earlier for smaller than for larger stimuli (see, e.g., Jakobson \& Goodale, 1991). Thus, if the results show longer or shorter movement duration and deceleration time with respect to the size of the distractor, an influence of the distractor size on the reaching kinematics of the action toward the target could be suggested. Furthermore, if the results show a smaller or larger maximum grip aperture and/or a later or earlier occurrence of maximum grip aperture with respect to the size of the distractor, inferences regarding the influence of the distractor size on the kinematics of the grasp component could be advanced. Given the nature of the task to be performed upon the distractor, we predicted that only the dependent measures concerned with the grasp component would be affected by the experimental manipulations.

\section{Data Analysis}

For each dependent variable, the $\mathrm{BC}, \mathrm{VC}$, and $\mathrm{C} 0$ conditions were analyzed separately in a $2 \times 3$ repeated measures analysis of variance (ANOVA) with hand (right or left) and condition (BC, VC, or $\mathrm{C} 0$ ) as the within-subjects factors. We will refer to these conditions as control conditions. Then the four conditions in the distractor task were analyzed in a $2 \times 4$ repeated measures ANOVA with hand (right or left) and condition ( $\mathrm{C} 0, \mathrm{C} 1, \mathrm{C} 2$, or $\mathrm{C} 3)$ as the within-subjects factors. The corresponding post hoc test was used to compare mean values whenever the main effects from the ANOVA were significant. Probability threshold was set at $p=.05$ for all the tests.

\section{RESULTS}

\section{Reaching Component}

The total movement duration and the deceleration time were not affected by the experimental manipulations. No significant main effect of hand or condition and no hand $X$ condition interactions were revealed from comparing the control conditions (BC, $\mathrm{VC}$, and $\mathrm{C} 0$ ) or from comparing the distractor task conditions $(\mathrm{C} 0, \mathrm{C} 1, \mathrm{C} 2$, and $\mathrm{C} 3$; see Figures 2A and 2B).

\section{Grasping Component}

Amplitude of maximum grip aperture. As regards the amplitude of maximum grip aperture, when the control conditions (BC, $\mathrm{VC}$, and $\mathrm{C} 0$ ) were compared separately, there was no significant main effect of hand $[F(1,7)=$ 

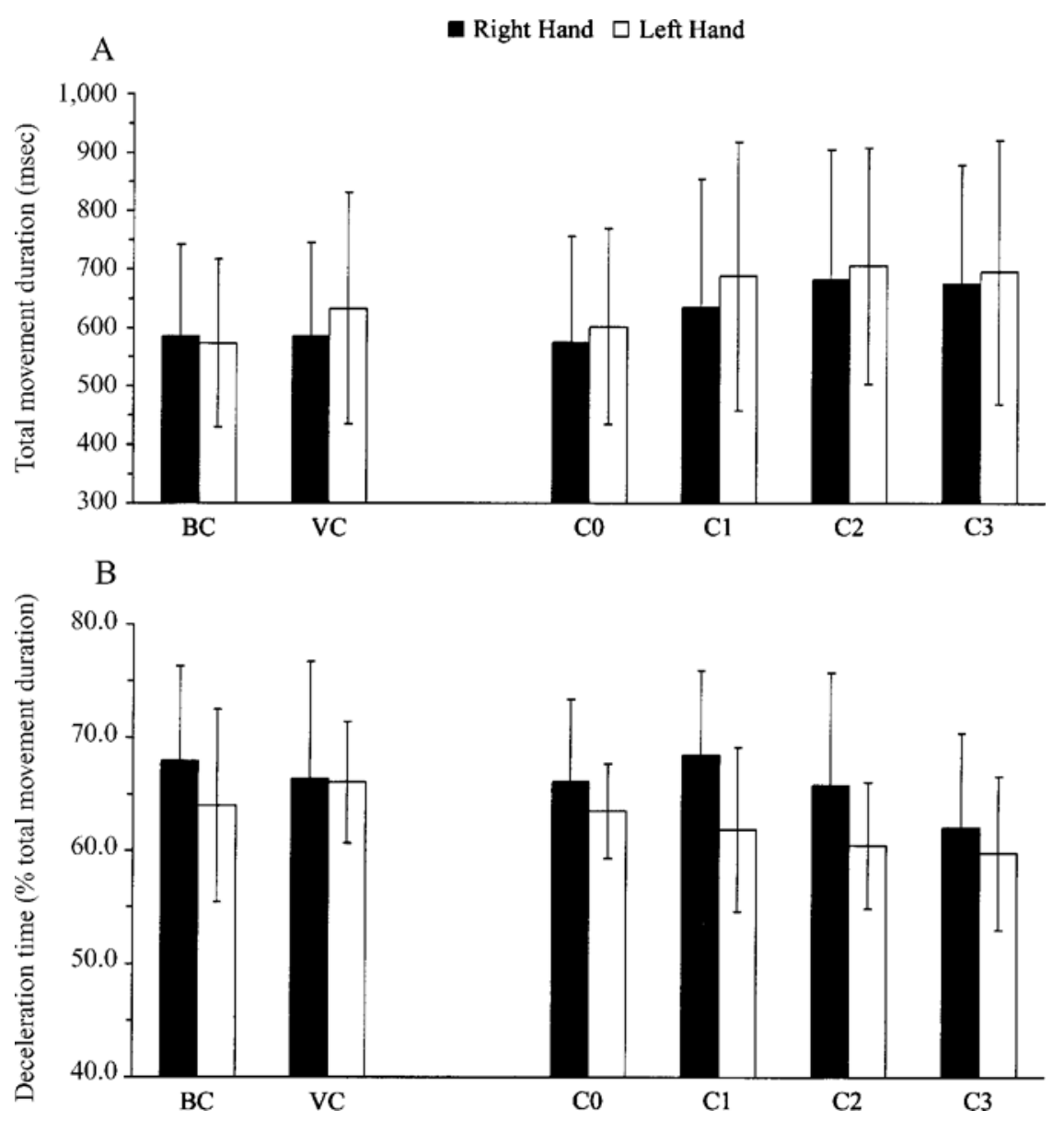

Figure 2. Mean values of total movement duration in milliseconds (panel A) and deceleration time expressed as a percentage of movement duration (panel $B$ ) for right and left hands. Error bars are plus and minus one standard deviation. BC, baseline control condition; VC, vibrating control condition; $\mathrm{C}$, control condition randomized with the different distractor sizes; $\mathrm{C} 1$, distractor smaller than target; $\mathrm{C2}$, distractor same size as target; $\mathrm{C3}$, distractor bigger than target.

$0.29, p>.05]$ or of condition $[F(2,14)=2.42, p>.05]$. Moreover, no significant $[F(2,14)=1.19, p>.05]$ hand $\times$ condition interactions were revealed (see Figure $3 \mathrm{~A}$ ).

When the distractor task conditions $(\mathrm{C} 0, \mathrm{C} 1, \mathrm{C} 2$, and $\mathrm{C} 3$ ) were compared, a significant main effect of condition was revealed $[F(3,21)=22.71, p<.001]$. Post hoc contrasts for the main effect of condition, using a Bonferroni adjustment, showed significant differences in the amplitude of maximum grip aperture between $\mathrm{C} 1$ and $\mathrm{C} 0, \mathrm{C} 1$ and $\mathrm{C} 2$, and $\mathrm{C} 1$ and $\mathrm{C} 3$ (all $p \mathrm{~s} \leq .005$ ). However, the grip aperture in $\mathrm{C} 3$ (distractor bigger than target) was not significantly different from that found in $\mathrm{C} 2$ (distractor same size as target; see Figure 3A).

Conversely, there was no significant main effect of hand $[F(1,7)=0.65, p>.05]$ and no significant interactions between the type of condition administered and the hand used $[F(3,21)=0.70, p>.05]$ among the distractor task conditions. Furthermore, to emphasize any hand interference effects, separate statistical analyses were conducted for the right and the left hands. These analyses revealed a significant effect of condition for the right hand $[F(3,21)=$ $16.18, p<.001]$, as well as for the left hand $[F(3,21)=$ $14.18, p<.001]$. Post hoc Bonferroni tests showed significant differences in the mean values of the amplitude of maximum grip aperture between $\mathrm{C} 1$ and $\mathrm{C} 0, \mathrm{C} 1$ and $\mathrm{C} 2$, and $\mathrm{C} 1$ and $\mathrm{C} 3$ (all $p \mathrm{~s}<.05$ ), but no difference between $\mathrm{C} 2$ and $\mathrm{C} 3(p>.05)$.

Time to maximum grip aperture. The time to maximum grip aperture, expressed as a percentage of total movement duration, showed no significant main effects of hand $[F(1,7)=1.85, p>.05]$ and condition $[F(2,14)=0.52$, $p>.05]$ nor any hand $\times$ conditioninteractions $[F(2,14)=$ $2.30, p>.05]$ in the comparisons between the control conditions (BC, VC, and C0; see Figure 3B).

Conversely, comparisons among the distractor task conditions $(\mathrm{C} 0, \mathrm{C} 1, \mathrm{C} 2$, and $\mathrm{C} 3)$ revealed a significant main effect of condition $[F(3,21)=17.85, p<.001]$. Post hoc contrasts showed significant differences in the mean val- 

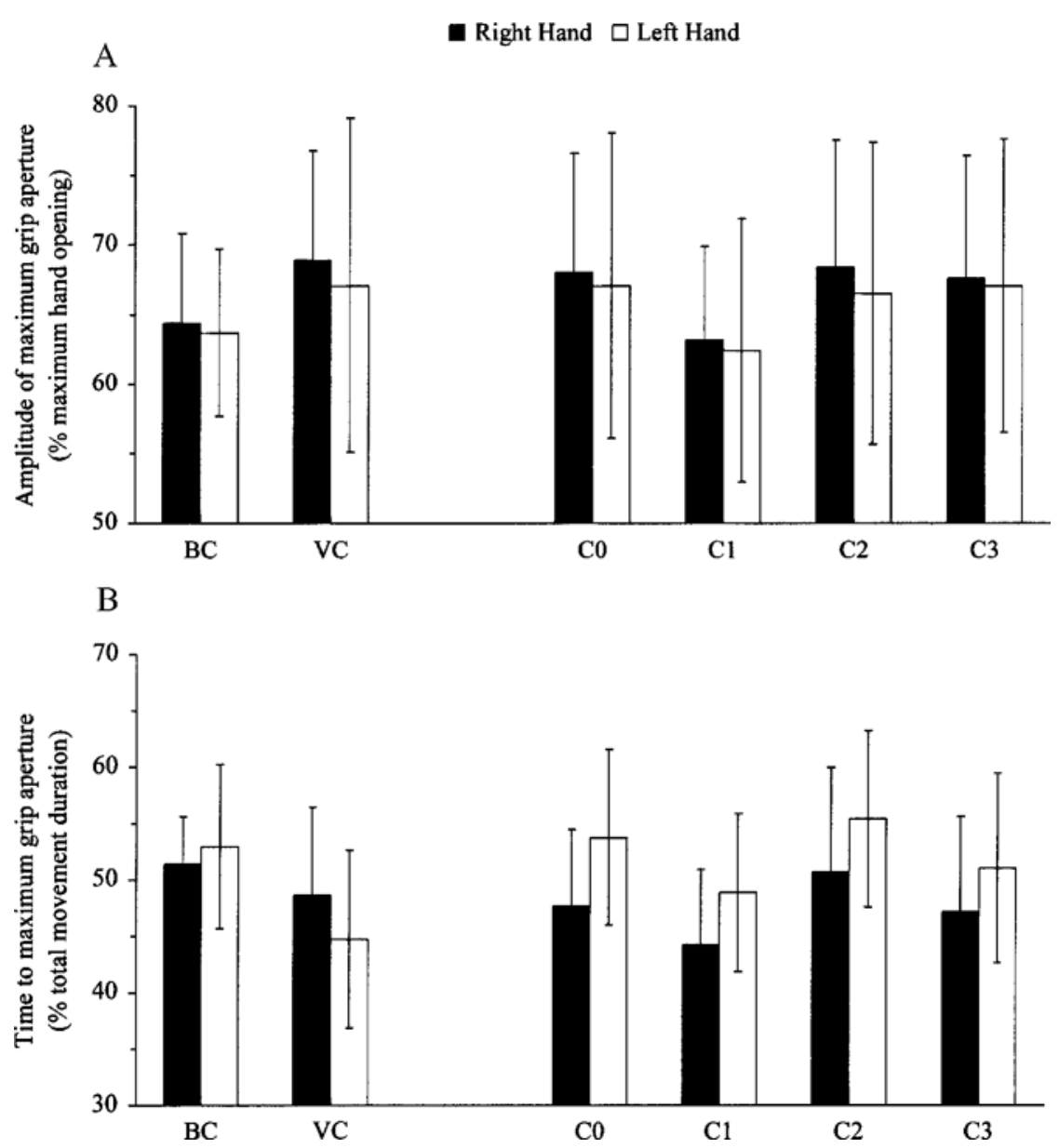

Figure 3. Mean values of amplitude of maximum grip aperture expressed as a percentage of participant's maximum hand opening (panel $A$ ) and time to maximum grip aperture expressed as a percentage of total movement duration (panel $B$ ) for the right and left hands. Error bars are plus and minus one standard deviation. BC, baseline control condition; VC, vibrating control condition; $\mathrm{CO}$, control condition randomized with the different distractor sizes; C1, distractor smaller than target; C2, distractor same size as target; C3, distractor bigger than target.

ues of the time to maximum grip aperture between $\mathrm{C} 1$ and $\mathrm{C} 0, \mathrm{C} 1$ and $\mathrm{C} 2$, and $\mathrm{C} 2$ and $\mathrm{C} 3$ (all $p \mathrm{~s}<.05$ ). There was no significant main effect of hand $[F(1,7)=1.99, p>$ $.05]$ and no significant interactions between hand and condition $[F(3,21)=0.49, p>.05]$ among these conditions. Separate analyses conducted for the right and the left hands revealed a significant effect of condition for both the right hand $[F(3,21)=10.17, p<.001]$ and the left hand $[F(3,21)=5.42, p<.01]$. Post hoc contrasts showed significant differences in the mean values of the time to maximum grip aperture between $\mathrm{C} 1$ and $\mathrm{C} 0$, and between $\mathrm{C} 1$ and $\mathrm{C} 2(p \mathrm{~s}<.05)$. There was no difference between $\mathrm{C} 2$ and $\mathrm{C} 3(p>.05)$.

\section{DISCUSSION}

In this study, we investigated how the kinematics of a hand reaching to a visual target are influenced by haptic and proprioceptive input from an unseen distractor manipulated in the nonreaching hand. In accordance with classical descriptions of the reach-to-grasp movement (Jakobson \& Goodale, 1991; Jeannerod, 1981), the index finger and the thumb begin to shape during the transport of the hand toward the target, and the maximum grip aperture is influenced by object size. Most important, we found that when the distractor was smaller than the target, the amplitude of maximum grip aperture was smaller and the time at which maximum grip aperture was reached was earlier than in the conditions in which the size of the distractor was not taken into account (BC and VC) or altered (C0). Similar patterns were observed for both the left and the right hands. Such response attraction between the two hands has been previously reported by Kelso, Southard, and Goodman (1979) in their study of coordination in bimanual reaching and, more recently, by Jackson, Jackson, and Kritikos (1999) in a bimanual reach-to-grasp task. 
In line with our predictions, the present findings demonstrate selective interference effects of the distractor on the grasping component of the reaching hand, suggesting that the interference elicited by proprioceptive and haptic information from the distractor selectively affects the grasping component of the reaching hand. This is in agreement with previous accounts of interference in the visual modality, where it has been found that distractor objects can selectively influence the reaching or the grasping components of a reach-to-grasp movement to a target, depending on whether the location or the size of the distractor is manipulated (Bonfiglioli \& Castiello, 1998). A similar study by Haggard (1991), investigating the effect on the timing of a right-hand grasp of the requirement to perform a grasp with the left hand, showed that the same left-hand grasp perturbation had no effect on reaching.

Gentilucci et al. (1998) found that manipulation of the distractor with the left hand did not produce any interference effect on the reach-to-grasp movement performed by the right hand. Thus, interference effects were confined to the left reaching hand when the distractor was manipulated by the right hand. In contrast, we found that both reaching hands were affected by the presence of the distractor. Possible explanations for our finding that the interference effect occurred with both hands relate to distractor and target locations, to active grasping of the distractor, and to the divisions of the corticospinal tracts.

We believe that the coincident locations of distractor and target stimuli in our experiment can largely account for our results. This argument finds support in the work of Graziano and Gross (1993), who demonstrated maximal response to temporal and coincident visual and tactile inputs, suggesting that sensorimotor integration appears to be enhanced when sensory stimuli are located in a coordinate system centered on a relevant body part.

A vibrating-distractortask was also included in this experiment to separate passive from active grasping. Interference effects were obtained only when the distractor was actively grasped. The importance of movement between stimulus and touch (active touch) in eliciting better tactile perception has been demonstrated previously (Chapman, 1994; Gibson, 1962). Besides, it is worth noting that in the protocol in which the distractor was actively grasped, both hands performed the same type of grasping action. This implies that an identical motor program was engaged for both hands, and hence, we can rule out any interference effect due to competition between the concurrent execution of two different motor programs, which was the case in other studies (Gentilucci et al., 1998; Kelso et al., 1979). Therefore, the interference effect we observed was most likely to have been relevant to the input from the distractor itself.

Another possible explanation is related to the pathways of descending information to the effectors. It is known that the ventral corticospinal tract projects bilaterally, whereas the majority of the fibers of the lateral tract cross over to the contralateral side of the spinal cord and are principally involved in the control of distal musculature of the fingers and, to a lesser extent, the proximal musculature of the arm (Ghez \& Krakauer, 2000). Moreover, sensory information from the hand reaching the contralateral primary somatosensory cortex can also reach the ipsilateral side via cortico-cortical pathways. On the basis of all this anatomical evidence, it can be claimed that each hemisphere has the potential to exert dominant control over reaching and grasping movements with the contralateral hand. Further evidence that both hemispheres can have competence for scaling reaching and grasping components come from studies of patients with unilateral (right or left) brain damage (Hermsdörfer, Ulrich, Marquardt, Goldenberg, \& Mai, 1999).

A further aspect of our results was that the maximum grip aperture and the time to maximum grip aperture were not different when the distractor was bigger than (C3) and identical to $(\mathrm{C} 2)$ the target. This finding probably denotes a ceiling effect that could reflect a strategy manifested to prevent extreme joint angle. Fingers have considerable biomechanical interdependence, in the sense that individuated finger movements occur by coordinated action of multiple muscles. This biomechanical issue and active postural maintenance might be controlled simultaneously from the primary motor cortex, as has been documented by Sanes and Schieber (2001). It is likely that the neural elements within the motor cortical neural network for the hand continually recombine while forming new output properties, to produce normal and/or adaptive motor behavior.

In conclusion, there is compelling evidence from our results for a neural cross-talk between the hand actively manipulating the distractor and the hand reaching to grasp the target. The interference effects observed are likely to be a consequence of neural processing of the proprioceptive and tactile information relevant to distractor size.

\section{REFERENCES}

Bonfiglioli, C., \& Castiello, U. (1998). Dissociation of covert and overt spatial attention during prehension movements: Selective interference effects. Perception \& Psychophysics, 60, 1426-1440.

Burke, D., Gandevia, S. C., \& MAcefield, G. (1988). Responses to passive movement of receptors in joint, skin and muscle of the human hand. Journal of Physiology, 402, 347-361.

CASTIE Llo, U. (1996). Grasping a fruit: Selection for action. Journal of Experimental Psychology: Human Perception \& Performance, 22, 582-603.

Castiello, U. (1999). Mechanisms of selection for the control of hand action. Trends in Cognitive Sciences, 3, 264-271.

Chapman, C. E. (1994). Active versus passive touch: Factors influencing the transmission of somatosensory signals to primary somatosensory cortex. Canadian Journal of Physiology \& Pharmacology, 72, 558-570.

Cordo, P., Carlton, L., Bevan, L., Carlton, M., \& Kerr, G. K. (1994). Proprioceptive coordination of movement sequences: Role of velocity and position information. Journal of Neurophysiology, 71, 1848-1861.

D'Amico, M., \& Ferrigno, G. (1992). Comparison between the more recent techniques for smoothing and derivative assessment in biomechanics. Medical Biology \& Engineering Computing, 30, 193-204. Deubel, H., Schneider, W. X., \& PAProtta, I. (1998). Selective dorsal and ventral processing: Evidence for a common attentional mechanism in reaching and perception. Visual Cognition, 5, 81-107. 
DoYle, M. C., \& WALKer, R. (2002). Multisensory interactions in saccade target selection: Curved saccade trajectories. Experimental Brain Research, 142, 116-130.

DrIVER, J., \& S PENCE, C. (1999). Cross-modal links in spatial attention. In G. W. Humphreys, J. Duncan, \& A. Treisman (Eds.), Attention, space and action: Studies in cognition neuroscience (pp. 130-149). Oxford: Oxford University Press.

Gentilucci, M., Daprati, E., \& Gangitano, M. (1998). Haptic information differentially interferes with visual analysis in reaching-grasping control and in perceptual processes. NeuroReport, 9, 887-891.

Ghez, C., \& Krakauer, J. (2000). The organization of movement. In E. R. Kandel, J. H. Schwartz, \& T. M. Jessell (Eds.), Principles of neural science (pp. 653-673). New York: McGraw-Hill.

Gibson, J. J. (1962). Observations on active touch. Psychological Review, 69, 477-491.

Graziano, M. S., \& Gross, C. G. (1993). A bimodal map of space: Somatosensory receptive fields in the macaque putamen with corresponding visual receptive fields. Experimental Brain Research, 97, 96-109.

HAGGARD, P. (1991). Task coordination in human prehension. Journal of Motor Behavior, 23, 25-37.

Hermsdörfer, J., Ulrich, S., Marquardt, C., Goldenberg, G., \& MAI, N. (1999). Prehension with the ipsilesional hand after unilateral brain damage. Cortex, 35, 139-161.

JACKSON, G. M., JACKSON, S. R., \& KritiKos, A. (1999). Attention for action: Coordinating bimanual reach-to-grasp movements. British Journal of Psychology, 90, 247-270.

JAKOBSON, L. S., \& GoOdALE, M. A. (1991). Factors affecting higherorder movement planning: A kinematic analysis of human prehension. Experimental Brain Research, 86, 199-208.
JEANNEROD, M. (1981). Intersegmental coordination during reaching at natural visual objects. In J. [B.] Long \& A. [D.] Baddeley (Eds.), Attention and performance IX (pp. 153-168). Hillsdale, NJ: Erlbaum.

Johansson, R. S., \& Cole, K. J. (1994). Grasp stability during manipulative actions. Canadian Journal of Physiology \& Pharmacology, 72, 511-524.

Johnson, K. O., Yoshioka, T., \& Vega-Bermudez, F. (2000). Tactile functions of mechanoreceptive afferents innervating the hand. Journal of Clinical Neurophysiology, 17, 539-558.

JOLICÄUR, P. (1999). Restricted attentional capacity between sensory modalities. Psychonomic Bulletin \& Review, 6, 87-92.

Kelso, J. A. S., Southard, D. L., \& Goodman, D. (1979). On the coordination of two-handed movements. Journal of Experimental Psychology: Human Perception \& Performance, 5, 229-238.

SANES, J. N., \& SCHIE BER, M. H. (2001). Orderly somatotopy in primary motor cortex: Does it exist? NeuroImage, 13, 968-974.

S PenCE, C., PAVAni, F., \& Driver, J. (2000). Crossmodal links between vision and touch in covert endogenous spatial attention. Journal of Experimental Psychology: Human Perception \& Performance, 26, 1298 1319.

STEIN, B. E. (1998). Neural mechanisms for synthesizing sensory information and producing adaptive behaviors. Experimental Brain Research, 123, 124-135.

TIPPE R, S. P., HowARD, L. A., \& JACKSON, S. R. (1997). Selective reaching to grasp: Evidence for distractor interference effects. Visual Cognition, 4, 1-38.

(Manuscript received April 26, 2002; revision accepted for publication August 5, 2002.) 